\title{
Research on the Bearing Stability of High-Order Picking Vehicles
}

\author{
Changqing Cui ${ }^{1, a}$, Chunyan Yang ${ }^{2, b, *}$ \\ ${ }^{1}$ College of Mechanical Engineering, Baicheng Normal University, Baicheng, 137000, China \\ ${ }^{2}$ College of Computer Science, Baicheng Normal University \\ achqoo@163.com, b45665662@qq.com \\ *Corresponding author
}

Keywords: high—order picking vehicles; stability factors; Gravity Center

\begin{abstract}
: the user demand for electric vehicles is more and more intense, will make electric drive in green energy storage and the rapid growth of the vehicle. With the further development of logistics transportation, this paper analyzed the factors which influenced the bearing stability of high-order picking vehicles based on Matlab procedure, then it established dynamic equation through dynamic - static method. This paper analyzed the stability of the vehicles from height, trajectory, ways of driving and turning radius in order to constantly optimize the vehicle structure and control parameters. On the premise of ensuring its stability, it improved its mobility at the greatest extent. this paper proposes a high - level order pickers focus self-balancing technology, further improve the stability of the vehicle.
\end{abstract}

\section{Introduction}

At present China's economy continued to develop steadily, the storage and transportation and logistics industries has resulted in high-speed development period. The demand of the modernization of logistics distribution industry carrying vehicles even a record high, the market continued strong, has formed its long-term growth, in recent years, China's industrial vehicle production and sales year-on-year growth is very considerable. Electric storage vehicle of astonishing growth, according to the China machinery industry association vehicles branch of statistics, in 2010 China only electric storage vehicle sales of 29782 units, compared with 2009 increased about 60\%. With the further development of logistics transportation, combined with the strengthening of national environmental protection policy and the national environmental protection consciousness enhancement, the user demand for electric vehicles is more and more intense, will make electric drive in green energy storage and the rapid growth of the vehicle. At present, the domestic land resources scarce causing prices to soar, make people have to to the space to benefit the development of strategy, caused the domestic centralized stereoscopic warehouse is increasing. Three-dimensional storage is combined by computer control and manual control of tunnel type storage and handling equipment access a new warehouse the goods. Stereoscopic warehouse has become an important part of modern logistics system, has the economical use of land, reduce the loss of storage and transportation, improving logistics efficiency advantages. As the stereoscopic warehouse, growing high chosen vehicle will usher in huge opportunities for development.

The underpan structure of high—order picking vehicles is very compact, and the hoisting height is very high and the driving speed changes fast. Therefore, the vehicle is prone to rollover accident due to its high gravity. In order to ensure that vehicles can operate efficiently and safely in stereoscopic warehouse, we require that it must have good stability and maneuverability[1]. Stability means the ability to resist capsizing under self weight and external load. Maneuverability is embodied in the minimum turning radius of the vehicle and the suitable minimum roadway width of the warehouse[2]. Obviously, there is a contradiction between stability and maneuverability.

With the further development of logistics transportation, combined with the strengthening of 
national environmental protection policy and the national environmental protection consciousness enhancement, the user demand for electric vehicles is more and more intense, will make electric drive in green energy storage and the rapid growth of the vehicle [3-6]. High-level order pickers belongs to the category of storage vehicle, are essential to the order picking stereoscopic warehouse delivery vehicles, to reduce the labor intensity of workers, improve the working efficiency is of great significance. High - level Order Pickers chassis of compact structure, High hoisting height, speed changes quickly, so the vehicle center of gravity High and change fast, easy to overturn accidents [7]. This article will research on the comprehensive stability of the high-level order pickers, and put forward a kind of high-level order Pickers focus self-balancing technology, further improve the stability of the vehicle to ensure the mobility of the vehicle at the same time.

\section{Stability Factors of High-Order Picking Vehicles}

\subsection{Height Analysis.}

In order to analyze the stability factors of high-order picking vehicles, we have to establish physical model. But it is difficult to establish accurate model for the firm structure of high-order picking vehicle and its trajectory are relatively complex. Therefore, it needs to be simplified in a reasonable range.

Figure1 is the entity models of high-order picking vehicles. The whole vehicle is kept by horizontal support. The gravity of the whole vehicle(including vehicle itself, driver and goods) concentrates on the underpan's center line. The projection of the vehicle height on vehicle supporting plane is point E. During the movement, vehicle is affected by many forces, such as gravity, ground reaction force and inertial force. Vehicle stability includes static stability and dynamic stability. Static stability is one of the special case of dynamic stability, equaling to the state in which vehicle moves at a constant speed. So this paper only studies its dynamic stability. There are two ways of driving of high_order picking vehicles, one is straight driving and the other is cornering driving. But the load-carrying capability of vehicles is different under two ways, so this paper studies its stability respectively.

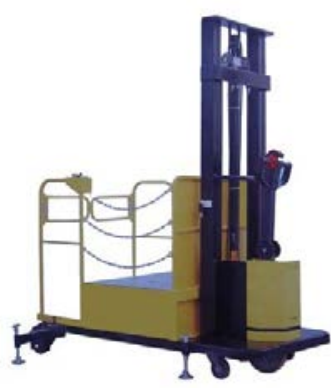

(seated type)

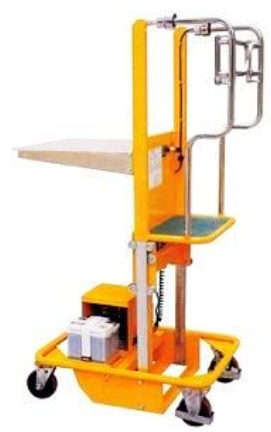

(stand-on type)

Fig.1 The entity models of high—order picking vehicles

\subsection{Analysis of Movement Locus.}

High-order picking vehicles operate on hard and smooth road, so it does not consider the influence of pavement deformation on stability. Therefore, it can rotate around one certain axis when vehicles overturn[8]. For three pivot vehicles, they are prone to lateral overturning during cornering driving. Its lateral overturning axis is ligature between the location of bearing wheel outside the cornering and the location of steering wheel. Take vehicles turning left as an example, it is easy to occur left lateral overturning with its movement locus, as shown in figure 2. 

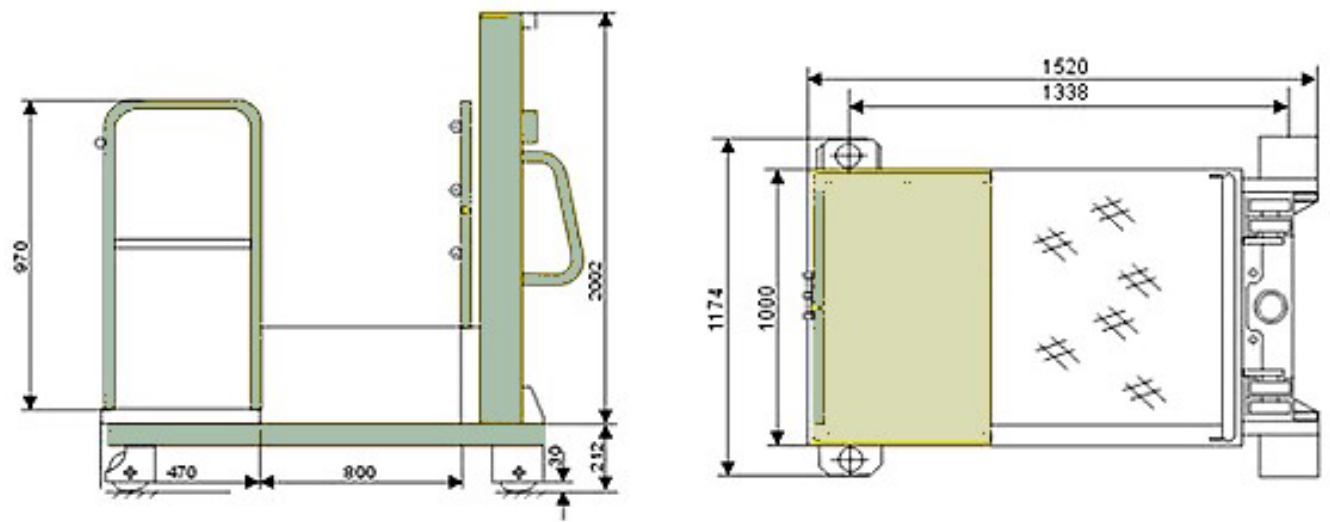

Fig.2 Left lateral overturning with its movement locus

\subsection{Ways of Driving.}

High—order picking vehicles are prone to occur longitudinal overturn during straight driving, so this paper is going to analyze its ways of driving under straight driving. Now take the state of vehicle accelerated speed as an example, we analyze its ways of driving and establish the model. The driving state of vehicle during moving is shown in figure 4 .

Suppose that vehicle can balance without capsizing under the joint action of the above several forces, we use D' alembert's principle to establish the dynamic equilibrium equations as follows[9]:

$$
\begin{gathered}
\sum F_{z}=N_{A}+N_{B}+N_{C}-m g=0 \\
\sum M_{B C}=N_{A} l_{A H}-m g l_{E F}+m a l_{D E}=0 \\
\sum M_{A H}=N_{B} l_{B H}-N_{C} l_{C H}-m g l_{E G}=0
\end{gathered}
$$

In this equation, $\mathrm{m}$ is vehicle's whole quality, including the weight of vehicle, goods quality and driver. a is vehicle's accelerated driving speed, $\mathrm{h}$ is the height from vehicle's gravity D to load bearing surface. NA,NB and NC are the counter-forces of ground to vehicle's front wheel, left rear wheel and right rear wheel respectively. $\sum f_{z}$ is the joint forces when vehicles are in the vertical direction. $\sum M B C$ is the resultant moment of all the forces to axis $\mathrm{BC} ; \sum M A F$ is the resultant moment of all the force to axis AF.

Now suppose that vehicles can maintain balance and stability under the existing structure and motion parameters, we calculate the counter-force of ground to three pivots NA, NB and NC, then regard these three forces as the index when measuring the stability of picking vehicles. If the counter-force NA, NB and NC are all positive values and bigger than a safe value under all motion conditions, then it proves that the dynamic stability of the vehicle is good. If the counter-force is close to zero in one motion condition, then it proves that vehicle is in the critical state of overturning and the stability is bad. If the counter-force is less than zero, then it proves that the suppose that vehicle can maintain balance is false, and vehicles are bound to instability and capsize under this motion state.

\subsection{Turning Radius.}

High-order picking vehicles are easy to occur lateral overturning in cornering driving, so this paper is going to analyze its turning radius in this working condition. Here, we take vehicle turning left as an example to establish mechanical moder [10]. As shown in figure 3 and figure 4, with the change of driving wheel's steering angle, moment turning center and turning radius of vehicles also changes. Now suppose driving wheel's steering angle is $\theta$ in a certain moment, and the 
instantaneous turning center of vehicle is point $O$. Suppose under the joint action of the forces, vehicles can maintain balance during cornering driving. Then analyze its dynamics and establish the dynamic equation, as shown in equation (4) to equation (11).

$$
\begin{gathered}
\sum F_{z}=N_{A}+N_{B}+N_{C}-m g=0 \\
\sum M_{A C}=N_{B} l_{M N}+\left(F_{g}^{n} \cos \beta+F_{g}^{t} \sin \beta\right) h-m g l_{E M}=0 \\
\sum M_{M N}=-N_{A} l_{A M}+N_{B} l_{B N}+N_{C} l_{C M}+\left(F_{g}^{t} \cos \beta-F_{g}^{n} \sin \beta\right) h=0 \\
l_{E H} \operatorname{arccot} \alpha+l_{G H}-l_{A G} \operatorname{arccot} \theta=0 \\
\alpha+\gamma-\beta-90^{\circ}=0 \\
F_{g}^{t}=m a_{t}=m v / t \\
F_{g}^{n}=m a_{n}=m \omega^{2} R \\
l_{E H} \\
\sin \left(\arctan \left(l_{E H} /\left(L_{1} \cot \theta-l_{G H}\right)\right)\right)
\end{gathered}
$$

In these equations, $\sum F z$ is the joint forces when vehicles are in the vertical direction. $\sum M_{A C}$ is the resultant moment of all the forces to overturning axis AC. $M_{E F}$ is the resultant moment of all the forces to axis EF. $F_{g}^{t}$ is the tangential inertial force when vehicle turns. According to equation (9), at is the tangential accelerated speed of vehicle's gravity, $v$ is the speed of the center; $F_{g}^{n}$ is the centrifugal force when vehicle turns. According to equation (10), an is the centrifugal accelerated force of vehicle's gravity, w is the angular speed when vehicle rotates around the instantaneous center $\mathrm{O} . \mathrm{R}$ is the turning radius of vehicle's gravity, whose size is related with the driving wheel's steering angel. Through kinematic analysis of vehicle turning process, turning radius can be calculated as equation (11).

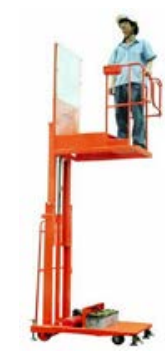

Fig.3 Stand-on type

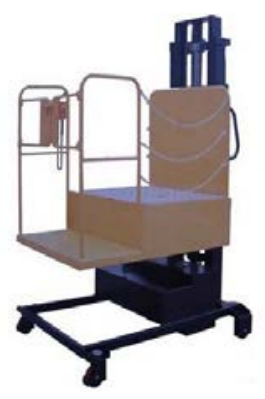

Fig.4 Seated type 


\section{Analysis of Stability on Mechanic Matlab}

In order to verify the above dynamic model, we use the designed and developed high-order picking vehicles as an example to analyze its stability based on mechanic Matlab. From equation (1) to equation (11), we know that during vehicle movement process, the size of the counter-force of ground to three pivots NA, NB and NC is closely related with vehicle's structure, speed, accelerated speed and load ,etc. Through the solution of the above kinematic equations and mechanic equations, we can know the influence of various parameters on the dynamic stability of high-order picking vehicles, so as to optimize the structural design and control strategy.

The self-weight $\left(m_{1}\right)$ of high-order picking vehicle developed in this paper is $3900 \mathrm{~kg}$, rated load (QC) is $1500 \mathrm{~kg}$, the quality of the driver is $80 \mathrm{~kg}$, wheel base $\left(L_{1}\right)$ between the front pivot and back pivot is $1537 \mathrm{~mm}$, span $\left(L_{2}\right)$ between left pivot and right pivot is $1200 \mathrm{~mm}$, load center distance is $60 \mathrm{~mm}$, maximum lifting height $\left(H_{\max }\right)$ is $9000 \mathrm{~mm}$. The accelerated time $(\mathrm{t})$ is $3 \mathrm{~s}$ when vehicle starts at the maximum speed $(V \max )$ in any time(t) in any lifting height. The braking time is calculated according to industry standard of warehousing vehicle, that is to say, the maximum braking distance of a vehicle can not surpass $1.5 \mathrm{~m}$.

No matter in the condition of no-load or full load, the minimum value of center-force of ground to three pivots NA, NB and NC is all more than $6000 \mathrm{~N}$, which proves when the lifting height $(\mathrm{H})$ is $1.8 \mathrm{~m}$, the vehicle's lateral stability is good during cornering driving, and it is not likely to occur capsizing. Similarly, through the analysis of the process of turning left at the typical lifting height with the scope of $\mathrm{H}$ ( $\mathrm{H}$ is 0.6 to $5.4 \mathrm{~m}$ ), we can get the minimum counter-forces of ground to pivots an various heights, as shown in table1.

Table 1 minimum counter-forces of ground to pivots when turning left

\begin{tabular}{c|c|c|c|c|c|c}
\hline $\begin{array}{l}\text { Lifting } \\
\text { height[m] }\end{array}$ & \multicolumn{3}{|c|}{ No-load } & \multicolumn{3}{c}{ Full load } \\
\hline & NAmin [N] & NBmin [N] & NCmin [N] & NAmin [N] & NBmin [N] & NCmin [N] \\
\hline 0.6 & 20317 & 5966 & 6583 & 12467 & 15325 & 17014 \\
\hline 1.2 & 20268 & 6320 & 6587 & 12468 & 15307 & 16682 \\
\hline 1.8 & 20211 & 6728 & 6631 & 12449 & 15594 & 16505 \\
\hline 2.4 & 20146 & 7201 & 6741 & 12415 & 16117 & 16477 \\
\hline 3.0 & 20076 & 7709 & 6914 & 12370 & 16814 & 16602 \\
\hline 3.6 & 20005 & 8225 & 7149 & 12317 & 17621 & 16879 \\
\hline
\end{tabular}

\section{Conclusion}

For the research on the stability of high-order picking vehicles, it currently widely adopts the front wheel angle step input to examine the transient and steady state response of high-order picking vehicles under the open loop control. But it has deficiency. When the range of structural parameter is large, A point on the root locus can represent a curve of the front wheel angle step response, with clear, intuitive and simple advantages. This paper first uses the root locus method in domestic to study the handling stability of high — order picking vehicles and gets the following conclusions:

(1) The influence of speed on limo trains is big. As the speed increases, the steering stability reduces significantly, or even instability.

(2) After the traction of high-order picking vehicles, the system damping ratio reduces significantly and stability margin reduces greatly.

(3) When analyzing the factors that influence the stability of high-order picking vehicles, we should pay attention to control other variables.

(4) The totational inertia of high-order picking vehicles is small and the distance from pin-connected joint to tractor rear axle is short, thus it helps to improve the handling stability of 
high—order picking vehicles.

\section{References}

[1] Makris, P.A,and Giakoumakis, I.G.k-interchange heuristic as an optimization procedure for material handling applications. Journal of Applied Mathematics. 2003

[2] Kim Byung in, Heragu Sunderesh S, Graves Robert J, St Onge Art. Clustering-based order-picking sequence algorithm for an automated warehouse. International Journal of Production Research. 2003

[3] Fujikawa Kazuhiro Fujii Takanori.Order picker truck. Japan, 2006 -076727. 2006

[4] Fabrizio Dallari,Gino Marchet,Marco Melacini. Design of order picking system[J]. The International Journal of Advanced Manufacturing Technology. 2009 (1-2)

[5] Janssen M.C,Verbeek R.J.D,Volgenant A.On scheduling a single machine with resource dependent release times. Computers \& Operations Reserch. 2011

[6] VR Muppani,GK Adil.Efficient formation of storage classes for warehouse storage location assignment: a simulated annealing approach. Omega. 2008

[7] De Koster R,Le Duc T,Roodbergen K J.Design and control of ware house or derpicking: A literature review. European Jou-rnal of Operational Research. 2007

[8] Park, B.C,Foley, R.D,Frazelle, E.H.Performance of miniload system with two-class storage. IIE Transactions. 2005

[9] DE KOSTER R, LE-DUC T, ROODBERGEN K J.Design and control of warehouse order picking: A literature review. European Journal of Operational Research. 2007

[10] Jane C.C, Laih Y.W. A clustering algorithm for item assignment in a synchronized zone order picking system. European Journal of Operational Research . 2005 